\title{
Beiträge zur Flora des Gömörer Komitates.
}

\author{
Von Nikolaus v. Szontagh.
}

\begin{abstract}
„Non fingendum aut excogitandum, sed videndum est, quid natura faciat aut ferrat." Baco Verulanus.
\end{abstract}

Wenn wir auch einige Quellen anführen können, die Angaben zur Flora des Gömörer Comitates enthalten, so müssen wir doch, um unbefangen und gerecht zu sein, bekennen, dass in botanicis diese Gegend bis jetzt fast eine terra incognita war; denn auch das sehr wenige, was Kitaibel und Wahlenberg in seinen Werken anführen, sind Genersich's und Ma ucksch's Verdienste, was ferner Reuss's „Kvetna slovenska ${ }^{1}$ ) enthält, ist theils Compilation aus den vorerwähnten und aus Flora muranyensis Manuskriple, theils beruhen die eigenen Daten aus der Umgebung von Röce, wo er als Arzt praktizirte, nicht auf kritisch-diagnostischer Grundlage und lassen so in vielen Fällen einen unüberwindlichen Zweifel zurück. Fábry's „Rimaszombat viránya “2) will ich gar nicht erwähnen; wir vermissen ja in ihr die lateinische Nomenklalur und finden nur die ungarischen Trivialnamen, mit denen man nach Gutdunken dies oder jenes bezeichnen kann.

Ich will nun in einige Gegenden aus dem nordwestlichen Theile dieses Komilates, die ich im August des Jahres 1864 durchstreilte, botanisch eingehen, und beginne gleich im Norden an der Grenze des Zipser Komitates in der pittoresken Sztracenaer Schlucht.

Der Königsberg (Kiralyhegy Králová hola) bildet mit seinen Voralpen eine hohe Mauer, die das Zipser vom Gömörer Komitate trennt. Nicht seine Höhe $\left(6134^{\prime}\right)$ zeichnet ihn vor den anderen Karpatenspitzen aus, sondern seine natürliche Lage und Gestalt, die ihm mit Recht den Namen des Königsberges gab. Während nämlich die Spitzen der Centralkarpaten, die ihm an Höhe nahestehen, die wilde Zerrissenheil kennzeichnet, bildet diese von den höheren Alpen isolirte, breitschulterige Bergmasse eine symmetrisch formirte Kuppel, die theils grünende Wiesen, theils dichte Waldungen decken, und dio nur an einigen Stellen aus Gneis bestehende Felsengruppen zu Tage fördert. Mithin ist diese Alpe ein vollkommenes Analogon der deutschen Alpen, an denen man bis zur Schneelinie die blühendsten Almen trifft. Was die Flora des Königsberges anbelangt, verweise ich auf Wahlenberg, Maucksch, Kitaibel, Genersich, besonders aber auf Aug. v. Kubinyi's Kiràndùlás Pohorellàròl a Kiràlyhágóra ${ }^{3}$ ) vorgetragen in der Versammlung ung. Aerzte und Naturforscher vom Jahre 1842.

\footnotetext{
1 Slavische Flora.

2) Flora von Rimaszombath.

3) Ausflug von Pohorella auf den Königsberg.
} 
Der Königsberg sendet gegen Osten zwei parallellaufende Aeste, deren eiñer im Zipser, der andere im Gömörer Komitate sich hinzieht. Zwischen beide eingebettet wälzt sich in wilder Hast der Göllnitz-Fluss, an dessen krystallenem Grunde die behenden Forellen spielend sich ergötzen. $Z u$ beiden Seilen begleiten seinen Lauf hier und da hohe Felsenwände, die an einigen Stellen sich so sehr nähern, dass sie nur noch der mit Meisterhand gebauten Strasse Raum lassen. Bald berühren sie sich, der Felsenkessel wird abgesperrt und nun muss der Fluss unterirdisch sich Bahn brechen und der Baumeister durch einen Tunnel sich verhelfen.

Aus seinem engen Bette bricht er zuweilen mit grossem Getöse hervor, in einen weiteren Kessel mündend, wo er in leisem Gemurmel grünende Wiesen benetzt. Das todte Steingerippe bildet übrigens nicht überall continuirlich verlaufende Felsengruppen, Wände oder Kämme, sondern nur dort, wo es aus Trias und Neocomkalk besteht. Hier entschleiert es dann seine nackten blendend weissen Flächen oder bildet vereinzelte Riesenstatuen, an denen hie und da Lärchenbaum-Kolonien prangen. Auf den genannten Kalkfelsen deren Spalten und Rissen wächst: Salix caprea L., Campanula rocundifolia L., Polemonium caeruleum L., Calamintha Acynos Clairv., Sedum album L., Saxifruga Aizoon L., Alsine laricifolia Whlbg., Ribes petraeum Wulf,, Aconitum Anthora L., Thesium intermedium S chrad., Arabis arenosa Scop. und alpina L. Am Ufer der Göllnitz und auf den Wiesen der Kessel wuchern in grosser Menge Senecio subalpinus L. und nemoralis L., Echinops sphaerocephatus L., Gentiana Amarella L., Geum urbanum L., Verbascum thapsiforme L. und andere. Die Wälder bestehen meist aus Fagus sylvatica L., gemischt mit Pinus Abies L. und P. Picea L., Acer campestre L. und Pseudoplatanus L. und Sorbus aucuparia L. Hier treffen wir Daphne Mezereum L., Knautia sylvatica Dub., Convallaria majalis L., Majanthemum bifolium L., Phyteuma spicata L., Rubus Idaeus L., Digitalis grandiflora L., Polygala amara L., Melampyrum sylvaticum L. und nemorosum L. Der aus Gneis und Glimmerschiefer bestehende westliche Theil des Sztracenaer Thales ist besonders ausgezeichnet durch einige seltene Pflanzenspecies wie durch die Cimicifuga foetida L., Anacamptis pyramidalis Rich., Gymnadenia conopsea R. Br., Orchis globosa L. und latifolia L., Gladiolus imbricat us L., Senecio Jacobaea L. und nemoralis L., Juniperus communis L., Avena pubescens $\mathrm{L}$. und flavescens $\mathrm{L}$.

Verlassen wir nun den Göllnilz-Fluss, der in östlicher Richtung seinen Lauf fortsetzt und lenken wir unsere Blicke gegen Süden auf den aus Glimmer und W erfnerschiefer bestehenden Dobschauer Wald. Kaum hatten wir dessen Anlöhe erreicht, als sich ein geräumiger Kessel unter unseren Füssen öffnet, in welchem die Bergstadt Dobschau eingebettet liegt. Der östliche 'Theil des Waldes besteht zumeist aus Fagus sylvatica L., Carpinus Betulus L., Corylus Avellana L., Betula pubescens Ehrh. und Populus nigra, unter welche Majanthemum bifolium D C., Hieracium praealtum L., Melam- 
pyrum nemorosum L., Genista tinctoria L., Hypericum quadrangulum L. und perforatum L., Dianthus deltoides L., Polystichum Filix mas Rth. und Galium Mollugo L. eingestreut sind. Lichte Stellen, trockene Triften sind bald ganz mit Vaccinium Myrtillus L. und Calluna vulgaris Sal. bedeckt, bald mengen sich unter sie Erigeron acre L., Carlina acaulis L., Avena flavescens L., Hieracium Pilosella L., Phleum pratense L., Trifolium montanum L., Coronilla varia L., Galium verum L. und Trifolium arvense L. An grasigen Plätzen und steinigen Orten finden wir Lepigonum rubrum $W \mathrm{hlb}$. Anchusa officinalis L., Galeopsis Tetrahit L., Silene inflata L., Filago arvensis L., Potentilla argentea L., Betonica officinalis L., Scleranthus annuus L. und Thesium intermedium Schrd. In der nächsten Umgebung von Dobschau wuchern am Rande des Sajó-Flusses Mentha aquatica L und sylvestris L., Leonurus Cardiaca L., Polygonum Hydropiper L., Senecio vernalis W. K., Cirsiuin.eriophorum S cop., Prunus Padus L., Chelidonium majus L., Euphrasia Odontites L, und officinalis L., Potentilla anserina L., Myosotis intermedia L nk. und palustris Wth., Alysma Plantago L., Lychnis flos cuculi L. und Verbascum Thapsus L. Auf Wiesen, Aeckern, waldigen Stellen unter Gebüsch begegnen wir überall auf Triaskalk Anthemis Cotula L. und arvensis L., Matricaria Chamomilla L., Trifolium repens L., arvense L. und pratense L., Linaria vulgaris Mill., Lappa major Gärtn., Sisymbrium officinale Scop., Achillea millefolium L., Hyoscyamus niger L., Bromus arvensis L., Polygonum aviculare L., Anthriscus oulgaris Pers., Aegopodium Podagraria L., Carum carvi L., Chenopodium vulvaria L. und Botrys L., Urtica urens L. und dioica L., Amaranthus Blitum L., Malva rotundifolia L., Potentilla argentea L., Carduus crispus L., Rosa canina L., Crataegus Oxyacantha L., Berberis vulgaris L. und Anagallis arvensis L. Etwas weiter entfernt von Dobschau fand ich auf Werfnerschiefer um Gabbro in Nadelwäldern bestehend aus Pinus Picea L., Abies L. und Larix L., Buchenwäldern auf Feldern und im Gebüsch Cirsium eriophorum L., Salvia verticillata L., Cineraria campestris Rtz., Potentilla argentea L., Carduus crispus L., Dianthus deltoides L., Sambucus racemosa L., Anthemis tinctoria L., Erigeron canadensis L., Solidago Virga aurea L., Linum catharticum L., Potentilla repıans L., Lychnis vespertina $S$ ibth., Anchusa officinalis L., Campanula glomerata L., Bupleurum folcatum $\mathrm{L}$. und Verbascum nigrum $\mathrm{L}$.

Von Dobschau aus fliesst der Sajò-Fluss in südlicher Richtung auf Glimmerschiefersubstrat zwischen bald niedrigere, bald höhere Anhöhen eingebettet, die meist mit Fagus sylvatica L. bewaldet sind, in welchem Populus nigra L., Betula pubescens Ehrh., Corylus Avéllana L., Alnus glutinosa L. und Quercus pubescens W. Kolonien eingesprengt sind. Hier fand ich Galium verum L., Anthyllis vulneraria L., Genista tinctoria L., Cytisus nigricans L., Inula britannica L., Verbena officinalis und Vicia Cracca $\mathrm{L}$.

In der Csetneker Gegend, welche der ausgebreiteten Tabakkultur wegen allbekannt isi, notirte ich von der nächsten Umgebung 
von Csetnek, die Grauwacken, Aluvium, Thon- und WerfnerschieferFormation zeigt, folgende Pflanzen: Zea Mays L., Nicotiana Tabacum L., Vitis vinifera L., Prunus Armeniaca L., Persica vulgaris Mill., Polygonum aviculare L., Solanum Dulcamara L., Datura Stramonium L., Xanthium strumarium L. und spinosum L., Arenaria serpyllifolia L., Rhamnus cathartica L., Lycium barbarum L., Morus alba L., Malva rotundifolia L., Althaea officinalis L., Artemisia vulgaris L., Epilobium angustifolium L. und Dodonaei Vill., Lythrum Salicaria L., Chenopodium Botrys L. Nordwestlich von Csetnek fand ich bei Szlabos auf Glimmerschiefer Artemisia Absinthium L., Lotus corniculatus L., Lycium barbarum L., Epilobium angustifolium L., Melilotus macrorrhiza Prs., Malva rotundifolia L., Althaea officinalis L., Polystichnm Filix mas Rlh., Poa compressa L. und annua L., Plantago media L., Salvia verticillata L., Sambucus Ebulus L. Bei Jolsoa sah ich auf Triaskalk Dypsacus sylvestris Mill., Lactuca Scariola L., Echinospermum Lappula Lhm., Cirsium eriophorum Scop., Fagus silvatica L., Quercus pubescens W. und Betula pubescens E hrh.

Derjenige Gebirgsast, weIchen wir schon oben am rechten Ufer des Gollnilz-Flusses antrafen, sendet beim Berge Hrona unweit seiner Ausgangsstelle vom Königsberg einen aus Triaskalk bestehenden Zweig gegen Südwest, der bei Murány jene spitzen Anhöhen und schroffen Felsengruppen bildet, auf deren einem die Murányer Burgruine steht. In floristischer Beziehung zeigen sie viel Ashnlichkeit mit der Kalkflora des Sztracenaer Thales. Besteigen wir den Murányer Schlossberg von seiner Südseite, so gelangen wir gleich am Fusse desselben in einen dichten Buchenwald, der höher oben mit Pinus Abies L., Picea L. und Larix L., Corylus Avellana L., Betula pubescens Ehrh., Fraxinus excelsior L., Acer campestre L. und Pseudoplatanus L. sich mengt. Die hier und auf lichten grasigen Plätzen bcobachteten Pflanzen sind die folgenden: Daphne cneorum L., Bellidiastrum Michelii Cass., Centaurea montana L., Sambucus racemosa L., Cynanchum Vincetoxicum L., Teucrium montanum L. und Botrys L., Siler trilobum Scop., Sisymbrium strictissimum L., Cotoneasier vulyaris L indl., Inula Oculus Christi L., Spiraea ulmifolia Scop., Cytisus biflorus L'Henri und ciliatus Whbg., Geranium robertianum L., Euphorbia epithymoides L., Eupatorium cannabinum L., Hypocrepis comosa L., Cypripedium calceolus L., Orchis militaris L. und globosa L., Corallorrhiza innata Rich., Carex ornithopoda Willd., Polygonum viviparum L., Digitalis grandiflora L., Festuca ovina L., Andropogon Ischaemum L., Valeriana dioica L., Dypsacus sylvestris L., Senecio Jacobaea L., Cineraria lungiflora J c q., Cirsium erisithales Scop., Tragopogon pratensis L., Campanula glomerala L., Galium sylvaticum L., Sambucus Ebulus L., Origanum vulgare Lam., Prunella grandiflora L., Echinospermum Lappula Lhm., Cynoglossum officinale L., Cuscuta europaea L., Verbascum thapsiforme S chrd., Antirrhinum najus L., Primula officinalis L., Archangelica officinalis $\mathrm{H} f \mathrm{fm}$., Sempervivum tectorum 
L., Aquilegia vulgaris L., Arenaria serpyllifolia L., Lychnis diurna Sibth. und Rhamnus cathartica $\mathrm{L}$.

Haben wir nach mühsamen steilem Gange den Wald hinter und unter uns, so gelangen wir zu den Trümmern einer auf einem schroffen spitzen Felsen gebauten Burg, welche nur noch schwache Ángedenken längst vergangener Zeiten sind. Doch ihre verwitterten Mauern und Trümmer, sowie Felsenspalten und Schluchten sind nicht uninteressant für den Botaniker, denn seine Mühe wird hier durch den Fund so mancher Lieblingspflanzen reichlich belohnt und zwar durch Thesium alpinum L., Campanula carpatica J cq., Polemonium caeruleum L., Corthusa Matthioli L., Soldanella alpina L., Aconitum Anthora L. und Napellus L., Sedum album L., Saxifraga Aizoon L., Siler trilobum S cop., Alsine laricifolia $\mathrm{W}$ h b g., Helianthemum vulgare Gärtn., Erysimum cheiranthoides L., Hieracium saxatile Jc q., Poa alpina L., $\alpha)$ collina, Anthericum ramosum L., Calamintha alpina Lam., Polygala amara L, Potentilla aurea L. und Lactucu perennis $\mathrm{L}$.

Noch will ich jener Pflanzen gedenken, die ich südwestlich von Murany theils auf der Fortsetzung des oben erwähnten Triaskalkstreifens, theils auf Werfnerschiefer bei Tisżòc und nicht weit von hier im sogenannten Furmanetzer Thale beobachtet habe. Diese sind: Petasites officinalis Mch., Sedum album L., Campanula Scheuchzeri Vill., Fagus sylvatica L., Ulmus campestris L., Populus nigra L., Sorbus aria Cntz., Pinus Abies L. und Picea K., Alnus glutinosa $\mathrm{G} n \mathrm{t}$., Leontodon hastilis L., Xanthium spinosum L., Erigeron acre L., Echinops sphaerocephalus L., Euphrasia Odontites L. und Potentilla reptans $\mathrm{L}$.

Wien, im Dezember 1865.

\section{Stotternheim bei Erfurt.}

Von J. Ch. Mühlefeld.

Stotternhein ist ein Dorf und gehört jetzt zum Grossherzogthume Sachsen-Weimar-Eisenach; vor circa 60 Jahren war es Erfurl, seiner Marktstadt einverleibt und stand mit ihr unter dem Krummstabe des Erzbischofs von Mainz. Es liegt zwei Stunden nördlich von Erfurt, an der Chaussee nach Sommerdar und an einem kleinen Bache, der südöstlich vom Dorfe entspringt und nach kurzem Laufe zwischen. Stotternheim und dem benachbarten Nöda in die sogenannte schmale Gera, einen Nebenarm der Gera, füllt. Auf der Karte finden wir es unter $51^{\circ} 3^{\prime}$ n. Br. und $28^{\circ} 42^{\prime}$ w. L. Die Höhe des Ortes über dem Ostseespiegel haben wir nicht genau ermilleln können. Nach den neuesten Messungen des preussischen Generalstabes (1859) liegt das 